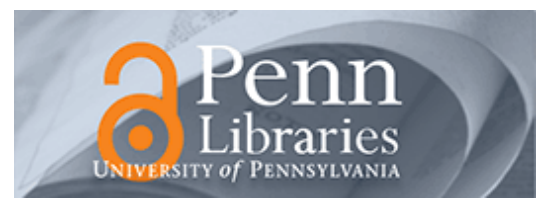

University of Pennsylvania

ScholarlyCommons

Operations, Information and Decisions Papers

Wharton Faculty Research

$9-2009$

\title{
Impact of Workload on Service Time and Patient Safety: An Econometric Analysis of Hospital Operations
}

Diwas S. KC

Christian Terwiesch

University of Pennsylvania

Follow this and additional works at: https://repository.upenn.edu/oid_papers

Part of the Health and Medical Administration Commons, and the Health and Physical Education Commons

\section{Recommended Citation}

KC, D. S., \& Terwiesch, C. (2009). Impact of Workload on Service Time and Patient Safety: An Econometric Analysis of Hospital Operations. Management Science, 55(9), 1486-1498. http://dx.doi.org/10.1287/ mnsc.1090.1037

This paper is posted at ScholarlyCommons. https://repository.upenn.edu/oid_papers/185

For more information, please contact repository@pobox.upenn.edu. 


\title{
Impact of Workload on Service Time and Patient Safety: An Econometric Analysis of Hospital Operations
}

\begin{abstract}
Much of prior work in the area of service operations management has assumed service rates to be exogenous to the level of load on the system. Using operational data from patient transport services and cardiothoracic surgery-two vastly different health-care delivery services-we show that the processing speed of service workers is influenced by the system load. We find that workers accelerate the service rate as load increases. In particular, a $10 \%$ increase in load reduces length of stay by two days for cardiothoracic surgery patients, whereas a $20 \%$ increase in the load for patient transporters reduces the transport time by 30 seconds. Moreover, we show that such acceleration may not be sustainable. Long periods of increased load (overwork) have the effect of decreasing the service rate. In cardiothoracic surgery, an increase in overwork by $1 \%$ increases length of stay by six hours. Consistent with prior studies in the medical literature, we also find that overwork is associated with a reduction in quality of care in cardiothoracic surgery-an increase in overwork by $10 \%$ is associated with an increase in likelihood of mortality by $2 \%$. We also find that load is associated with an early discharge of patients, which is in turn correlated with a small increase in mortality rate.
\end{abstract}

\section{Keywords}

health-care operations, productivity, service operations, optimal control of queues, quality

\section{Disciplines}

Health and Medical Administration | Health and Physical Education 


\title{
The Impact of Work Load on Service Time and Patient Safety: An Econometric Analysis of Hospital Operations
}

\author{
Diwas Kc, Emory University \\ Christian Terwiesch, The Wharton School, University of Pennsylvania
}

April 15, 2009

\begin{abstract}
Much of prior work in the area of service operations management has assumed service rates to be exogenous to the level of load on the system. Using operational data from patient transport services and cardiothoracic surgery - two vastly different healthcare delivery services - we show that the processing speed of service workers is influenced by the system load. We find that workers accelerate the service rate as load increases. In particular, a $10 \%$ increase in load reduces length of stay by 2 days for cardiothoracic surgery patients, while a $20 \%$ increase in the load for patient transporters reduces the transport time by half a minute. Moreover, we show that such acceleration may not be sustainable. Long periods of increased load (overwork) have the effect of decreasing the service rate. In cardiothoracic surgery, an increase in overwork by $1 \%$ increases length of stay by 6 hours. Consistent with prior studies in the medical literature, we also find that overwork is associated with a reduction in quality of care in cardiothoracic surgery - an increase in overwork by $10 \%$ is associated with an increase in likelihood of mortality by $2 \%$. We also find that load is associated with an early discharge of patients, which is in turn correlated with a small increase in mortality rate.
\end{abstract}

We are grateful to the cardiac anesthesiologists and executives at the teaching hospital where this study was conducted. We also thank the Management Science review team as well as Stefanos Zenios, Chris Lee, and Marcelo Olivares for their insightful and constructive comments on an earlier version of this paper. The authors can be reached at dkc@emory.edu and terwiesch@wharton.upenn.edu. 


\section{Introduction}

Over the last decade, most hospitals have witnessed a substantial increase in fixed costs, largely reflecting growing expenses for new technologies and liability insurance. Over the same period, hospitals also had to face a substantial decrease in per-case reimbursements, reflecting the transition from "fee for service" reimbursements to contractual reimbursements due to managed care. As a result of these two trends, hospitals have come under increasing pressure to operate at very high levels of utilization. From a macro perspective, high utilization is a desirable system property for a hospital and its employees, as it spreads the fixed cost over a larger volume of patients. However, recent research conducted with a more micro perspective (Green 2004) has demonstrated that operating at high levels of utilization has many operational implications, including long waiting times.

Most of these micro level models are based on queueing analysis (Green 2004, Smith Daniels et al. 1988). Such models analyze patient flows and in particular patient waiting times based on information about the care capacity of the process, the variability of its service times, and the behavior of a stochastic demand for care. A high level of utilization (a high level of demand relative to the available capacity) leads to a dramatic increase in wait times and - if waiting is not feasible due to the emergency of the case or due to a limited amount of space - a reduction in patient flow (i.e. the number of patients cared for in a unit of time). Collectively, queueing analysis in healthcare has emerged as an active area of research with a clear potential for impacting healthcare practice.

A central assumption in this existing literature is that the service time, i.e. the time it takes a resource to care for a patient, is independent of the state of the process including the current work-load. In this paper we show this might not always be the case. Consider the data shown in Figure 1. As a motivating preview to one of our results, the figure shows the relationship between the risk-adjusted ${ }^{1}$ length of stay of

\footnotetext{
${ }^{1}$ In the medical literature, the risk adjusted length of stay is computed by first determining how
} 
cardiothoracic surgery patients as a function of the work-load in the cardiothoracic surgery unit ${ }^{2}$ at the time of discharge. We observe a clear pattern indicating that the service time (duration the patient is in the unit) decreases with an increase in workload. The unit thus increases its throughput when it is busy. In other words, its level of care capacity seems to be adaptive to higher levels of work-load. From an empirical perspective as well as from the perspective of hospital management, the data shown in Figure 1 raises a set of interesting research questions. (1) What drives this increase in processing speed? Is the hospital simply discharging patients prematurely, or is there evidence that the same work gets done faster? (2) Are there any implications for the quality of care provided? (3) Can the resources in the hospital sustain this increased service rate or does there exist an effect of overwork?

Figure 1: Length of Stay as a function of Census

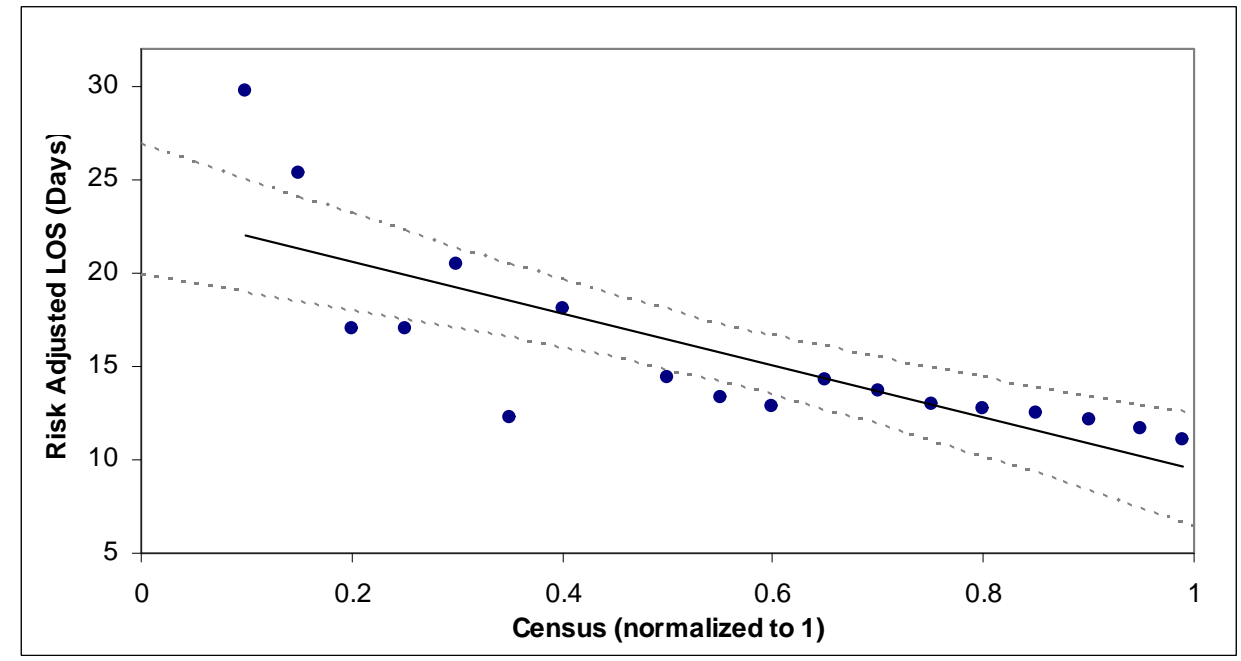

1. Census is defined as the number of patients in the cardiac unit at the time that a patient is admitted

2. Length of Stay (LOS) is the total number of days a patient spends at the hospital

3. Dashed lines represent $95 \%$ confidence intervals.

We address these three questions by conducting a detailed econometric analysis of two care processes in a major US teaching hospital. In particular, we look at process individual patient risk factors predict the length of stay, and then generating an expected length of stay for each patient based on their specific risk factors.

${ }^{2}$ The cardiothoracic surgery unit is the self-contained hospital unit that includes i) admissions ii) diagnostic testing (cath lab, ECG, etc.) iii) pre-operative care, such as prepping the patient for surgery iv) surgery, v) post-operative care (e.g. time in the ICU) and vi) discharge. 
and outcome data of some 3,000 cardiothoracic surgery patients. We measure the length of stay for each patient and relate it to a set of covariates, including current work-load and the cumulative fatigue, or workload burden on service workers. We address the alternative explanation of Figure 1 that the hospital simply discharges patients prematurely in two ways. First, we look at risk-adjusted mortality data to investigate how work-load and overwork lead to changes in mortality. Second, we also study another care process in the hospital that is not a medical process and does not provide the option of simply cutting the service time short at the potential cost of quality. In particular, we look at the service times of over 17,000 requests for patient transport and analyze how they change with work-load and the subsequent overwork.

This research design allows us to make the following three contributions. First, we measure the performance of hospital employees and show that employees adjust their service rates with changing levels of load. This is, to the best of our knowledge, the first empirical test of the insights obtained from the optimal queueing control literature. In cardiothoracic surgery we find that a $10 \%$ increase in load leads to a reduced length of stay (service time) of over 2 days (about 20\%). Similarly, we find that patient transporters speed up their tasks by half a minute (about $3 \%$ of service time) if load increases by $20 \%$. Second, our study investigates the impact of work-load as well as overwork on the quality of care, a relationship that is potentially a matter of life or death in a hospital. Overwork is defined as the excess work-load beyond an expected amount of work-load over a given period of time. Specifically, we establish that patients admitted to an overworked unit are associated with an increased risk of mortality. On average, a $10 \%$ increase in overwork is associated with a $2 \%$ increase in risk of mortality. Third, we show that while hospital employees can respond to increased work-load by increasing their productivity in the short run, such an acceleration in general is not sustainable. After a duration of exceptionally high work-load, employees are subject to the after-effects of overwork. This effect of overwork could outweigh the higher service rates discussed above. A sustained 
level of $1 \%$ above average load for a week in cardiothoracic surgery units leads to an average increase in length of stay of almost 6 hours $(2 \%)$.

If hospital employees are indeed capable of adjusting their service rate as a function of the work-load, this clearly has substantial implications for the management of care capacity. If service workers can adapt during periods of high work load by working faster, it may not be necessary to hire additional capacity during busy periods. Also, instead of relying on safety capacity to buffer against stochastic increases in demand, the hospital could rely on its staff's ability to temporarily accelerate their work. However, our empirical findings suggest additional managerial considerations that need to be made. Although such adaptive behavior from workers may appear desirable in the short run, one needs to also consider the quality and patient safety implications of such behavior. In addition, temporary worker speedup made come at the cost of future slow-down after the onset of fatigue. This could lead to a net total decline in performance. Decision makers should thus take into consideration the full set of possible implications of a temporary increase in service rates.

The remainder of this paper is organized as follows. In the next two sections, we present relevant literature and develop our hypotheses for a general model of service operations. We then operationalize our theory to the two hospital settings we study. Section 4 describes our research setting, the econometric model specification, and the results for our study of patient transporters. In section 5, we report the same information for the cardiac surgery setting. We conclude with discussions and future avenues for research in section 6 .

\section{Literature Review}

The Operations Research literature has created a number of tools that directly or indirectly relate to the management of care capacity and its utilization (see Green (2004) for an overview). At the strategic level, decisions need to be made with respect to 
sizing the care capacity. This includes choosing occupancy rates (e.g., Smith-Daniels et al. 1988, Huang 1995, Green and Nguyen 2001) and making staffing decisions (e.g., Aiken et al. 2002, Kwak and Lee 1997, Green and Meissner 2002). At the tactical level, decisions need to be made with respect to scheduling and sequencing cases (e.g., Gerchak et al. 1996) as well as with respect to allocating capacity to various demand types (e.g. Green et al. 2006). Much of this prior body of literature, however, assumes that the service rate is exogenous to the level of capacity utilization. In this paper, we present and validate a framework of service operations where workers vary their service rates with the state of the system. There also exists a significant body of literature dealing with optimal payment systems for health services, as reported by Newhouse (1996). Many of these studies (e.g. Fuloria and Zenios 2001) explore the effect of various types of payment arrangements that incentivize healthcare organizations into providing higher quality of services. Higher quality is often achieved only at a higher cost, of which workload and service rates are important contributors. This stream of literature seeks to examine how, in the presence of unobserved cost factors, appropriate incentives can still be provided to hospitals to induce higher quality. In addition to this general research on hospital operations, our analysis builds on two areas of prior research in operations management.

First we draw on the literature on the optimal control of queues. For example, Crabill (1972) and Bertsekas (2000) examine systems in which the service rate is adjusted dynamically as the queue length changes. ${ }^{3}$ Some of these models study the dynamic control of a single-server queueing system that has Poisson arrivals and exponentially distributed service times. There are costs associated with an increase in the queue length and in an increase in the service rate. The objective is to choose the optimal service rate that minimizes the average sum of these two costs over a given planning horizon. In other words, a key objective of this body of literature is

\footnotetext{
${ }^{3}$ Although previous work, e.g. Green (1984) model queueing system that involves multiple servers, as far as we are aware, there are no established optimal policies on service rate when multiple servers are involved.
} 
the development of service rate policies that effectively balance the costs of waiting with the costs of an accelerated service rate. Under relatively general assumptions, Stidham and Weber (1989) prove the existence of a stationary policy, i.e. one in which transition to a given state elicits the same service rate. Although closed form solutions for the optimal service rate as a function of queue length are not obtainable, George and Harrison (2001) develop a novel method for computing the optimal policy for the service time as a function of the queue length, subject to certain restrictions on the two cost functions. In such a setting, the optimal service rate is a non-decreasing function of the length of the queue. The intuition for the monotone policy is that working faster by a given unit rate has a bigger impact on total waiting cost when the queue is longer. In a similar vein, Berk and Moinzadeh (1998) also allow the service rate to vary, and normatively explore the impact of the option of a shorter service time on effective capacity. Even though the results in this body of literature are well established, there have been no empirical validations of this effect. We contribute to this line of research by providing explicit evidence of the adaptive behavior in two healthcare services. For both services, although the underlying waiting costs and service rate costs are not estimated, we show that service rates increase when the load on the system increases.

Our work also extends prior studies of the impact of production system design on the productivity of employees. For example, using lab-based experiments, Schultz et al. $(1998,1999)$ consider serial production systems in which adjacent workers in a serial assembly line can observe each others' productivity, as measured by inventory levels between them. A key insight from this work is that workers tend to work faster or slower depending on the work in process inventory. Our objective in this paper is to demonstrate using actual operational data from a field based study at a hospital, that healthcare delivery workers also demonostrate such adaptive behavior in response to the amount of work-load. In addition, the previous studies have not considered the aspects of fatigue that accompany service rate acceleration, or the 
impact on the quality of service. Our study augments the existing body of work to include the dimensions of fatigue and quality. Powell and Schultz (2004) show that when assembly line workers adapt to variations in load, they also improve the overall throughput of the system. One of the implications of our study is that the adaptive behavior of healthcare providers increases the overall process flow of patients from the hospital.

\section{Hypothesis Development}

Our theoretical framework is based on the relationships between service times, work load, overwork and service quality. All of these measures are defined for the discrete

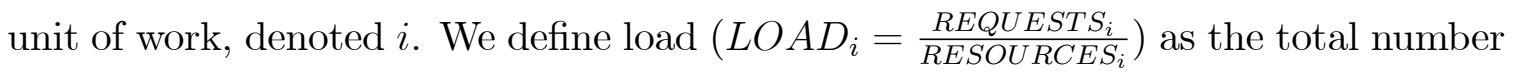
of requests or jobs (REQUESTS $\left.S_{i}\right)$ in the system divided by the total number of resources $\left(R E S O U R C E S_{i}\right)$ at that time that unit of work $i$ is in the system. In other words, $L O A D_{i}$ provides a measure of the level of utilization of the system's resources that is connected with the unit of work $i$.

We define $S V C T I M E_{i}$ to be the service time taken to process a request $i$. This definition of service time does not include any time spent waiting for the service to begin. Our hypothesis is that a higher work-load leads to a reduction in service time, i.e.

$$
\frac{\partial S V C T I M E}{\partial L O A D}<0
$$

Such a behavior can be rational from the worker's perspective if each service worker's utility is decreasing in the level of waiting time at a greater rate than the decrease in the utility associated with effort involved in obtaining a faster service rate, as theoretically established in the literature on the optimal control of queues.

Although productivity gains may be achieved in the short term as we hypothesize, high service rates may not be sustainable for longer periods of time. During periods of increased load, a worker may be motivated to work fast, but eventually fatigue effects 
may start to dominate, leading to increased service times. Early research in the field of Ergonomics (Cakir et al. 1980) has shown that as fatigue rises, productivity falls. Tanabe and Nishihara (2004) use lab experiments to study changes in productivity and find that even though people are highly motivated in short term experiments, they become tired and performance deteriorates over a longer time frame as fatigue kicks in. Likewise, a key finding in the studies by Caldwell (2001) and Setyawati (1995) is that fatigued workers exhibit diminished productivity.

In order to study the phenomenon above, we construct the measure $O V E R W O R K_{i, K}$, which we define to be an increasing function in the difference between the observed $L O A D_{i}$ and the average over $K$ units of time prior to the arrival of unit of work $i$ in the system. In other words, when a unit of work $i$ arrives at the system after a period of sustained levels of high $L O A D_{i}$ for $K$ units of time, our measure of OVERWORK $K_{i, K}$ will be high. We argue that this holds for a broad set of values of K used to estimate OVERWORK. Based on the discussions above we propose that the service time is increasing in the overwork. That is,

$$
\frac{\partial S V C T I M E}{\partial O V E R W O R K}>0
$$

We next consider the impact of the above effects of load, overwork and service time on the quality of service (QUALITY), which is of paramount importance in healthcare delivery. During periods of high $L O A D$, resources are more thinly spread out. We hypothesize that this decrease in the availability of resources can lead to a decline in quality. That is,

$$
\frac{\partial Q U A L I T Y}{\partial L O A D}<0
$$

Similarly, we argue that a patient who is admitted to an overworked unit has a higher likelihood of encountering a quality lapse, as service workers who are more fatigued are more prone to making mistakes. That is,

$$
\frac{\partial Q U A L I T Y}{\partial O V E R W O R K}<0
$$


Finally, we hypothesize that when service times are decreased (after controlling for patient specific factors), and patients are discharged early, this could have an adverse impact on the quality of care.

$$
\frac{\partial Q U A L I T Y}{\partial S V C T I M E}>0
$$

To test the hypotheses above, we chose two vastly different kinds of services - patient transportation and cardiothoracic surgery - at a major US teaching hospital. Patient transportation is, relative to other healthcare tasks, simple and the task of moving a patient from one part of the hospital to another is rather mechanical in nature. Typically each transport lasts less than half an hour. In sharp contrast, service workers in cardiothoracic surgery require advanced medical knowledge and extensive training. The individual tasks in cardiothoracic surgery are more complicated, and the average patient length of stay is around two weeks.

A study looking at patient transport alone might be dismissed as not being applicable to more medical and diagnostic processes. A study looking at cardiac care alone might be dismissed with the claim that patients are simply discharged prematurely as opposed to receiving care at a faster service rate. Replicating our research design across these two different care processes hence increases the generalizability of our findings. Below, we provide context-specific justifications for the hypotheses outlined above, followed by our findings for the two studies.

\section{The Patient Transport Study}

Patient transporters are hospital employees who perform the crucial role of taking a patient from one part of the hospital to another. The hospital that we study maintains a pool of between 2 and 26 transporters, depending on the time of day. When a patient is ready for transport, the nurse in charge of the hand-over submits an electronic request. The request then is placed in a queue to be processed by a dispatcher. When a transporter is available, the dispatcher assigns a transporter to a 
specific request. After a transporter arrives at the transport location, the transport process begins.

We operationalize the variables defined in the previous section as follows. REQUESTS is the total number of transport requests and RESOURCES $S_{i}$ is the number of transporters working on the shift at the time that request $i$ arrives. $L O A D_{i}$ is the fraction of transporters who were busy during the hour that transport $i$ was started. So if 5 out of 10 transporters were occupied at the time that service $i$ was rendered, $L O A D_{i}=50 \%$. Note that our definition of $L O A D_{i}$ corrects for anticipated increases in demand that were addressed by an increase in scheduled capacity. For example, the hours between 9 a.m. and 10 a.m. on a regular weekday, show 3 times more transport requests than there are between 9 p.m. and 10 p.m. However there are also $2 \frac{1}{2}$ times more transporters staffed during the busier period. The SVCTIME for each transport $i$ is the time between the patient leaving the starting location and arriving at the final destination. This does not include any waiting time for the transporter to arrive.

We define $O V E R W O R K_{i, K}$ at the level of the transporter, and the measure for $O V E R W O R K_{i, K}$ is computed only if the transporter performing service $i$ was on shift for each of the $K$ periods prior to the start of service $i$. Let $t(i)$ be the time at which unit $i$ arrives. To formalize the notion of overwork, we define $O V E R W O R K_{i, K}$, from time $t(i)-K$ up to time $t(i)$ as

$$
O V \operatorname{ERWORK} K_{i, K}=\frac{1}{N(K, i)} \sum_{j=i-N(K, i)}^{i-1}\left(L O A D_{j}-\overline{L O A D_{s(j)}}\right)
$$

where $\overline{L O A D_{s(j)}}$ is the average load over the entire shift $s$ and $N(K, i)$ is the number of service requests during the last $K$ periods up to $t(i)$. The $K$ periods are measured in units of hours. For example, suppose that the expected load during a certain shift is 4 requests per worker every hour. However, suppose that for a particular hour proceeding request $i(K=1)$, the load has consistently remained at 6 requests per worker during which 10 requests happened to have been processed. The overwork, 
$O V E R W O R K_{i, 1}$ associated with request $i$ would then equal $\frac{1}{10} \sum_{10}(6-4)=2$ requests per worker. In other words, the worker responsible for transporting request $i$ has already experienced an additional load of 2 requests on average over this time period.

An average transport lasts 13 minutes, and the average load on transporters is 0.76. Table 1 provides descriptive statistics of the key variables of concern. To achieve parallelism with the cardiothoracic surgery study, we sought out possible measures of quality in patient transport. In speaking to head of the patient transport services, we found that one source of error involves the patient being transferred to the wrong location. The other potential error is a lapse in adherence to specific protocols (for example, with handling of equipment and supplies). However, these errors are not captured and collecting this data is not currently feasible. Thus, although desirable, the quality implications of speedup are not estimated

\subsection{Econometric Analysis}

The variables SVCTIME and $L O A D$ do not take on negative values. Thus, we follow the commonly used approach of taking the natural logarithm of these variables to reduce the skewness in the distributions. We specify our regression model as:

$$
\log \left(S V C T I M E_{i}\right)=\beta_{0}+\mathbf{X}_{i} \boldsymbol{\beta}_{1}+\beta_{2} \log \left(L O A D_{i}\right)+\beta_{3} O V E R W O R K_{i, K}+\varepsilon_{i}
$$

where $\varepsilon_{i}$ is the mean zero error term. $\mathbf{X}_{i}$ consists of a set of variables that control for the underlying heterogeneity in patient characteristics and/or task characteristics. ${ }^{4}$ This includes indicators for the time of the day $(T I M E)$ and day of the week $(D A Y)$, which capture inter-temporal differences in elevator availability and hallway traffic as well as specific information about the transport. Transporters may be required to use additional pieces of equipment $(E Q U I P)$ along the way, including intravenous medication, oxygen, and other supplies. Transports also vary in mode $(M O D E)$;

\footnotetext{
${ }^{4}$ Unlike $L O A D, O V E R W O R K$ is emprically seen to have a zero-mean normal distribution, and the log-transformation does not produce a better model fit.
} 
some patients may require specialized telemetry beds, while others only need wheelchairs and transport beds. For example, a patient transport with a telemetry bed will take longer than with a wheel chair. For each transport $i$, we also correct for the person in charge of the transport $(N A M E)$, trip start $(S T A R T)$ and end $(E N D)$ locations, starting and ending locations for the transporter $(P A T H)$, and type of patient transported (TRIP_TYPE). Table A1 in the Appendix provides a list of variables and controls $\left(\mathbf{X}_{i}\right)$ for the econometric specification above.

As the load on the system increases in any given shift, the expected waiting times for transporters also tend to increase. Speeding up the transport time helps to somewhat mitigate the increase in waiting times. Thus, transporters (whose performance is constantly evaluated through a patient tracking system) have an incentive to speed up when the load on the system increases as outlined in (1). The coefficient of $\beta_{2}$ denotes the elasticity of service time with respect to load. A value of $\beta_{2}<0$ indicates that servers respond to high load by reducing the service time, providing support for (1).

To capture a potentially non-linear relationship between $L O A D$ and SVCTIME, we also created a categorical variable for $L O A D$ for values in the ranges 0 to $0.3,0.3$ to $0.5,0.5$ to $0.65,0.65$ to 0.8 , and 0.8 to 1 such that we had approximately similar numbers of observations within each range. We then estimated (6) above, replacing $\log \left(L O A D_{i}\right)$ with the categorical specification for $L O A D_{i}$.

Finally, as outlined in hypothesis (2), we expect $O V E R W O R K$ to be negatively correlated with the transport time $(S V C T I M E)$. Patient transport is a physically demanding task, and after a few hours of transporting patients, transporters may exhibit symptoms of tiredness and fatigue. Thus, a positive value of $\beta_{3}$ suggests that overwork leads to a longer service time, providing support for the hypothesis outlined in $(2)$. 


\subsection{Results}

Table 2 summarizes the results of estimating the above regression model with service time as a dependent variable based on a sample of 17,000 patient transports. We find that the elasticity of load on service time is -0.17 . This amounts to approximately $3 \%$ faster service on average $(p=0.02)$ for a $20 \%$ increase in LOAD. This statistically significant result provides strong support for our hypothesis that higher load leads to shorter service times.

Next, consider the effect of overwork. In estimating (6) above, we find that $K=4$ yields the best model fit. ${ }^{5}$ The regression results in Table 2 show that the coefficient for $O V E R W O R K\left(\beta_{3}\right)$ has a value of $0.09(\mathrm{p}$-value $=0.05)$. That, is a 0.1 unit increase in $O V E R W O R K$ (or the equivalent of a sustained level of 0.1 additional load above the expected load for $K=4$ hours) leads to an increase in service time by about $0.9 \%$. This lends support to hypothesis (2) that overwork leads to an increase in the service time in patient transport. Our result is consistent with our interviews with patient transporters and their management who reported based on their personal experience that transporters visibly slow down at the end of busier shifts. At any given point in time, a worker is subject to the effects of both existing load, and fatigue effects arising from sustained load in the immediate past. We find that the correlation between $L O A D$ and $O V E R W O R K$ is 0.295. Load and overwork have opposing effects, so at any given point in time, depending on the relative magnitudes of load and overwork, the net effect might be either a decrease or an increase in the service rate.

\footnotetext{
${ }^{5}$ Our estimations were performed with varying values for $\mathrm{K}$. The final value of $\mathrm{K}$ that was chosen yields the best maximum likelihood value.
} 


\section{The Cardiothoracic Surgery Study}

Unlike patient transport, cardiothoracic surgery is a highly specialized service involving numerous care providers. In our analysis, we observe the lengths of stay and quality measure of patients who pass through a single cardiothoracic surgery unit. We observe the admission and discharge dates for each patient, which are used to compute the patient length of stay as well as the daily census. The index $i$ identifies each unique patient admission. The associated service time $S V C T I M E_{i}$ is the total length of stay for the patient from the date of admission to the discharge date. REQUESTS $S_{i}$ measures the number of patients in the unit when patient $i$ was admitted (the census) and RESOURCES $S_{i}$ is the total bed capacity when patient $i$ arrives at the cardiothoracic surgery unit. In our period of study, the total bed capacity remained unchanged. $L O A D_{i}$ is thus defined to be the census divided by the total bed capacity at the time that patient $i$ is in the hospital. In our preliminary analysis (Figure 1), we looked at the effect of $L O A D_{i}$ at the time of admission. We also computed alternative measures of $L O A D_{i}$, including a measurement at the time of discharge, and at the midpoint of the patient's stay at the hospital. In addition, we also computed $L O A D_{i}$ over a nominal fixed length of stay for all patients. ${ }^{6}$ We find that all four measures of $L O A D_{i}$ have a very similar effect on service time (Appendix Table A4). For the remainder of this study, we compute $L O A D_{i}$ by using the daily average of load measured over the entire length of stay of patient $i$.

In contrast to our transport study, in the cardiac surgery study there exists no unique individual worker who performs all tasks related to a particular patient. Therefore, we estimate $O V E R W O R K_{i, K}$ at the level of the hospital unit using the daily load for $K$ days prior to the admission day for patient $i$. Let $d(i)$ be the date on which patient $i$ is admitted. We define $O V E R W O R K_{i, K}$, from time $d(i)-K$ up

\footnotetext{
${ }^{6}$ We thank the review team for suggesting the various measures of $L O A D$. The appendix includes our results for impact of the various measures of $L O A D$ on the service times.
} 
to time $d(i)$ as

$$
\text { OVERWORK } K_{i, K}=\frac{1}{N(K, i)} \sum_{j=i-N(K, i)}^{i-1} L O A D_{j}-\overline{D A I L Y_{-} L O A D_{d(j)}}
$$

where $\overline{D A I L Y \_L O A D_{d(j)}}$ is the average load in the unit on the date of admission of patient $j$, and $N(K, i)$ is the number of patient arrivals during the last $K$ periods up to $t(i)$. For example, a large positive value of $O V E R W O R K_{i, K}$ signifies that the unit has experienced high levels of load over the $K$ days of observation prior to the admission of patient $i$.

As indicated in the descriptive statistics (Table 3), we see that the average length of stay for a patient undergoing cardiothoracic surgery is 12.9 days. The standard deviation of 10.7 days indicates significant variability in length of stay, which is partly due to the heterogeneity amongst patients. The average load of 0.78 is comparable to the average load seen by transporters.

\subsection{Econometric Analysis}

We test hypotheses (1) and (2) using the econometric specification below:

$\log \left(S V C T I M E_{i}\right)=\gamma_{0}+\mathbf{Y}_{i} \gamma_{1}+\gamma_{2} \log \left(L O A D_{i}\right)+\gamma_{3} O V E R W O R K_{i, K}+\gamma_{4} M O N_{-} W E D+\varepsilon_{i}$

$\mathbf{Y}_{i}$ includes a set of variables that control for the underlying heterogeneity in patient characteristics, as well as temporal factors such as day of week. The patient population includes cardiac patients that vary widely in length of stay and risk levels. To account for cardiothoracic surgery specific factors that influence the $S V C T I M E_{i}$ and outcome, as measured by the occurrence of post-surgery mortality $\left(M O R T A L I T Y_{i}\right)$, we include several clinical pre-operative risk factors including age $\left(A G E_{i}\right)$, sex $\left(S E X_{i}\right)$, race $\left(R A C E_{i}\right)$, emergency status $\left(E M E R_{i}\right)$, and various specific medical co-morbidities, and complicating factors to correct for patient level heterogeneity. We use two commonly used medical estimates of patient-specific risk. 
The measure EUROSCORE $E_{i}$ is estimated on a 0 to 1 scale and captures the preoperative level of patient risk based on a number of individual patient risk factors. A similar risk score developed by the New York Heart Association $\left(C L A S S_{-} N Y H A_{i}\right)$ was also available for each individual patient. We also observe the type of procedure $\left(P R O C E D U R E_{i}\right)^{7}$ performed, as this has a significant bearing on the length of stay. For example, a patient will need a longer recovery time following a combined valve and bypass surgery compared to a single bypass surgery. We correct for temporal factors that could affect the length of stay (through staffing shortages during holiday season and on weekends for example) by using indicator variables denoting month $\left(M O N T H_{i}\right)$ and day of week $\left(M O N_{-} W E D_{i}\right)$ of admissions. Finally, we also observe incidences of a patient having to be re-intubated (RE_INTUBATED $\left.D_{i}\right)$. Re-intubation ${ }^{8}$ occurs if a patient is put on ventilator support for a second time. Tables A2 and A3 in the Appendix provide detailed definitions of all operational and medical variables.

Since hospitals often cite bed capacity as the primary reason for the inability to admit new patients, we believe that bed capacity utilization is the most significant driver of admission and discharge decisions, and ultimately determines a patient length of stay. In other words, when the system is busy, beds are in greater demand. Consequently, there is a pressure to discharge patients faster in order to free up bed capacity. The effect of an increase in load on reducing the length of stay thus makes hypothesis (1) appear tenable in the context of a cardiothoracic surgery unit. The coefficient of $\gamma_{2}$ denotes the elasticity of service time with respect to load. A value

\footnotetext{
${ }^{7}$ We did not observe individual surgeons involved in the procedures. However, each cardiothoracic procedure is highly specialized and is performed by either one or two surgeons. For instance, mitral valve procedures are operated by only one surgeon. Thus, PROCEDURE also serves as a proxy for the surgeon.

${ }^{8}$ Intubation is the placement of a flexible plastic tube into the trachea to protect the patient's airway and provide a means of mechanical ventilation. If a patient is intubated again (or reintubated), it is an indicator of increasing patient severity, and possibly longer length of stay.
} 
of $\gamma_{2}<0$ indicates that the unit respond to high load by reducing patient length of stay, providing support for the hypothesis outlined in equation (1).

To further characterize the relationship between $L O A D$ and SVCTIME, we created a categorical variable for $L O A D$ ranging from 0-0.6, 0.6 - 0.7, 0.7-0.8, 0.8 - 0.9, and 0.9 - 1 . We then estimated (6) above, replacing $\log \left(L O A D_{i}\right)$ with the categorical specification for $L O A D_{i}$.

Prior research investigating the performance of health workers has investigated the effect of worker fatigue on clinical decision making and outcome. In particular, fatigued and overworked medical residents and nurses have been observed to create more medical errors in diagnosis and treatment (e.g. Scott et al. 2006). For example, Gaba and Howard (2002) point out that most studies on fatigue show impairment of clinically relevant tasks. We argue that fatigue could impact the length of stay in two ways - either because the decision maker would like to take more time to make the discharge decision ${ }^{9}$, or because fatigued workers are more prone to making medical errors. We hypothesize that such errors lead to complications that call for additional rework, which would further lengthen a patient's stay. Hypothesis (2) is supported if fatigue leads to an increase in the patient's length of stay. Recall that $K$ is the duration of units of time over which high load brings about a noticeable amount of fatigue. The value of $K$ that yields the best model fit for specification (7) is chosen as the period of time over which $O V E R W O R K_{K}$ is estimated. The coefficient $\gamma_{3}$ captures overwork effects. A positive value of $\gamma_{3}$ suggests that a sustained period of high load leads to a longer service time, providing support for the hypothesis outlined in equation (2).

The prior medical literature relies on self-reported measures of fatigue. In this paper, we show that our objective, census-based measure of overwork also increases the length of stay. This suggests that overwork could be used as a proxy for a measure

\footnotetext{
${ }^{9}$ In discussions with medical staff, we noted that doctors are more likely to prescribe medical tests when discharge and diagnosis decisions become difficult.
} 
of the level of fatigue, where self-reported values are unavailable or biased.

We also examine whether staffing levels could affect the length of stay of patients. Although we do not directly observe the daily staffing levels in our data set, we note that medical care providers, including nurses, anesthesiologists, and residents typically work regular weekly schedules. Consequently, any variations in the level of medial staff are "seasonal" on a weekly basis. That is, the staffing level changes can be controlled for by simply accounting for the day of the week. In our preliminary analysis, we find that the number of staff does not vary greatly during weekdays. However, staffing levels are slightly lower during weekends. We also find that the average length of stay is slightly less than two weeks. This means that a patient admitted on a weekend would have stayed on average two weekends in the hospital, whereas a patient admitted early in the week would most likely have spent only one weekend. Given that the weekday staffing levels are higher than weekend staffing levels, the patient who ends up spending two weekends experiences more days with fewer support staff. Thus, by explicitly controlling for the day of week of admission, we account for the weekly schedule-related changes in the level of staffing that could drive the observed length of stay effects. In the econometric specification above, $M O N_{-}+W E D=1$ if a patient was admitted on either a Monday, Tuesday or Wednesday, and $M O N_{-} W E D=0$ otherwise. $\gamma_{4}$ estimates the effect of a weekend or near-weekend admission on increasing the length of stay.

We next consider the effect of load and overwork on the quality of service. In healthcare operations, medical outcome is commonly used as a measure of quality of service. Compared to patient transport, outcomes are much more important and also more accurately quantifiable in the case of cardiothoracic surgery. Our focus with respect to quality is to investigate if and to what extent process variables such as work-load and overwork are significant covariates when predicting mortality.

In this setting, a large body of medical literature has statistically analyzed variables that influence the risk-adjusted mortality score (Nashef et al. 2002, Kurki 2002, 
www.euroscore.org). Following a long line of medical research in cardiac surgery, the EuroSCORE model is one such statistical model that attributes a mortality score to a set of patient level risk factors. Specifically, the EuroSCORE model takes a number of medical covariates, such as such as gender, age, and medical conditions, as well as procedure specific attributes, such as the nature of the procedure, and links them to the binary outcome of mortality using a logit regression. That is, the EuroSCORE model is essentially a logistic regression model with the dependent binary variable as quality of care and the independent variables as the pre-operative and procedure-specific risk factors. The Appendix lists the set of independent variables used by the EuroSCORE model. In our analysis we the augment the EuroSCORE model to examine the effect of additional covariates such as load and overwork on the mortality rate.

We study two mechanisms in which process variables might affect mortality. First, work-load and overwork might impact the risk of mortality during the hospitalization of the patient. For example, Needleman $(2002,2006)$ found that a higher number of hours of care by registered nurses per patient is associated with better care. Aiken et al. (2002) report that higher patient to nurse ratios are linked with higher patient mortality and failure to rescue among surgical patients. Following this prior work, we argue that for intensive care patients such as those in a cardiothoracic unit, a decrease in the time that doctors and nurses have available on a per patient basis leads to an increase in risk-adjusted mortality during the hospitalization. Define the binary variable $M O R T A L I T Y_{-} I H_{i}$ such that MORTALITY $\_I H_{i}=1$ if the $i$-th patient died during the hospitalization and MORTALITY_IH $H_{i}=0$ otherwise.

To test hypotheses (3) and (4) using in-hospital mortality as a measure of quality, we augment the EuroSCORE model by including the variables $L O A D$ and $O V E R W O R K$ as additional covariates. We consider the effect of $L O A D$ and $O V E R W O R K$ on all post-operative in-hospital mortalities. This leads to the following logistic regression 
model:

$$
\operatorname{logit}\left[\operatorname{Pr}\left(\text { MORTALITY }_{-}{ }_{\text {IH }}\right)\right]=\mu_{0}+\mathbf{Z}_{i} \boldsymbol{\mu}_{1}+\mu_{2} L O A D_{i}+\mu_{3} O V E R W O R K_{i, K}
$$

where $\mu_{0}$ is the base-line rate of in-hospital mortalities. $\mathbf{Z}_{i}$ includes the 19 medical covariates that are used in the EuroSCORE model to predict patient mortality. ${ }^{10} \mathrm{~A}$ positive value of $\mu_{2}$ in (8) would provide support for the hypothesis outlined in (3), indicating that patients entering cardiac surgery at a time when the unit is highly utilized face a higher mortality risk. Likewise, a positive value of $\mu_{3}$ would provide support for the hypothesis outlined in (4), indicating that patients entering cardiac surgery at a time when the resources have been exposed to an extended period of high work-load (i.e. are overworked) face a higher mortality risk.

Second, process variables might also impact mortality after the hospitalization of the patient, i.e. the mortality of patients who have already been discharged. We use the post-discharge mortality as an additional measure of quality. Define the binary variable MORTALITY ${ }_{-} P D_{i}$ with MORTALITY ${ }_{-} P D_{i}=1$ if the $i$-th patient died within 30 days post-discharge and MORTALITY_PD $D_{i}=0$ otherwise. Just as we hypothesized for the in-house mortalities, we aim to analyze if an increase in load or the cumulative effect of overwork leads to an increase in probability of post-discharge mortality. Unexpected complications might be overlooked by a busy or overworked work-force.

In addition to validating (3) and (4), there exists another effect of process variables on mortality that is unique to the post-discharge mortality. A high work-load might induce the hospital to discharge patients early; this in turn might increase the odds of mortality. However, to examine the effect of early discharge on mortality rate, it is not enough to simply observe the relationship between mortality and length of stay. This is because a longer hospital stay could be associated with increased case severity and a higher likelihood of mortality. On the other hand, a shorter length of stay due

\footnotetext{
${ }^{10}$ Service time is not included in this empirical specification because for in-hospital mortalities, the patient discharge decisions and hence length of stay are not explicit decision variables.
} 
to an earlier discharge could lead to a lower quality of care, resulting in an increased likelihood of mortality. Our objective is to identify this second effect. In order to do so, we need to separate the confounding effect of severity of illness on the length of stay.

We do this by first computing the predicted length of stay for case $i, S V \widehat{C T I} M E_{i}$. Among cardiothoracic surgery patients, medical risk factors such as patient age, sex and various co-morbidities as well as procedure type are considered to be significant predictors of length of stay. We estimate this risk-based expected length of stay $\left(S V \widehat{C T I} M E_{i}\right)$ using such medical risk factors. We then compute the variable $E A R L Y D I S_{i}$ as the difference between the actual length of stay $\left(S V C T I M E_{i}\right)$ and the predicted length of stay $\left(S V \widehat{C T I} M E_{i}\right)$ :

$$
E A R L Y D I S_{i}=S V \widehat{C T I} M E_{i}-S V C T I M E_{i}
$$

The variable $E A R L Y D I S_{i}$ then captures changes in the length of stay caused by non-medical risk factors. In particular, we hypothesize that an increase in load leads to an early discharge. In order to establish an increase in load leads to an early discharge, we use the following econometric specification:

$$
E A R L Y D I S_{i}=\kappa_{0}+\mathbf{Y}_{i} \boldsymbol{\kappa}_{1}+\kappa_{2} \log \left(L O A D_{i}\right)+\tau_{i}
$$

where $\tau_{i}$ is the random error term. A positive value of $\kappa_{2}$ suggests that an increase in load leads to an early discharge. This in turn could impact mortality. By definition, early discharges only influence post-discharge mortality.

Next, to demonstrate that an increase in mortality occurs due to an early discharge, we use $E A R L Y D I S_{i}$ in a new logistic regression.

$$
\begin{aligned}
& \text { logit }\left[\operatorname{Pr}\left(\text { MORTALITY_PD } D_{i}\right)\right]=\eta_{0}+\mathbf{Z}_{i} \boldsymbol{\eta}_{1}+\eta_{2} L O A D_{i}+ \\
& \eta_{3} O V E R W O R K_{i, K}+\eta_{4} E A R L Y D I S_{i}
\end{aligned}
$$

Positive values for $\eta_{2}$ and $\eta_{3}$ suggest that load and overwork directly contribute to an increase in mortality. Positive values for $\kappa_{2}$ and $\eta_{4}$ suggest that load indirectly contributes to mortality by inducing early discharges. 


\subsection{Results}

We estimate our models based on a sample of 2740 patients corresponding to all admissions in our study period including the years 2003-2006. Table 4 summarizes the regression results with length of stay as a dependent variable. We find that the length of stay decreases when the load on the system increases. For example, as indicated by the estimation using models (1) and (2), a 10\% increase in load on average, leads to a shorter length of stay by $20 \%$. Given that the average length of stay is around two weeks, this amounts to a significant reduction in length of stay of almost two and a half days on average. However, the variation in $L O A D$ in cardiothoracic surgery is relatively low compared to transport service, as indicated by the standard deviations in the descriptive statistics. Thus, only a relatively small fraction of the sample experiences load related changes of more than one day. We also find that the effect of $L O A D$ on $S V C T I M E$ is increasing as the value of $L O A D$ increases.

We also observe the effect of overwork in cardiothoracic surgery. In estimating (7) above, we find that $K=7$ yields the best model fit. ${ }^{11}$ As Table 4 illustrates, a 0.01 unit increase in $O V E R W O R K_{K}$ leads to a $2 \%$ (6 hours) increase in the length of stay. Overall, we find that overwork has an important bearing on the performance of the cardiac unit and that high service rates cannot be sustained for longer periods of time, as postulated by the hypothesis in (2). In addition, we find that a weekday admission $\left(\gamma_{4}=0.09\right)$ is associated with a shorter length of stay. Specifically, a patient who is admitted on a weekend has a longer length of stay by about $9 \%$. One explanation for this is that staff levels are lower during the weekends. As a result, many services such as imaging, diagnostic testing and surgical services are curtailed. This means that a patient who is admitted close to a weekend is more likely to wait until the next weekday before full services can be rendered. In particular, non-scheduled patients

\footnotetext{
${ }^{11}$ Our estimations were performed with varying values for $\mathrm{K}$. The final value of $\mathrm{K}$ that was chosen yields the best maximum likelihood value.
} 
admitted through the emergency department also have to wait before they can be added to the surgical schedule. This has effect of increasing overall length of stay for patients who are admitted closer to a weekend. ${ }^{12}$

Now, we turn to the impact of the process variables on mortality and first examine the in-hospital mortalities (Table 5). We do find that overwork has a statistically significant effect $\left(\mu_{3}=3.53, \mathrm{p}\right.$-value $\left.=0.01\right)$, supporting the hypothesis outlined in equation (4) that patients admitted to an overworked unit are at increased risk of mortality. In particular, a $10 \%$ increase in $O V E R W O R K$ is associated with a $2.2 \%$ increase in mortality rate. ${ }^{13}$ This result is consistent with findings in the medical literature linking fatigue to a decrease in quality of care. However, our measure of fatigue (or overwork) is obtained from observed work-load, whereas the previous studies relied on self-reported measures from service workers. The effect of load is not statistically significant at the $10 \%$ level.

Next, we look at the results for the post-discharge mortality (Table 7). The coefficients $\eta_{2}$ and $\eta_{3}$ are not statistically significant at the $10 \%$ level, suggesting that overwork and load do not directly impact the post-discharge patient mortality. Thus, there is no support for the hypotheses indicated by (3) and (4) when tested on the post-discharge patient mortality. However, the coefficient for EARLYDIS $\left(\kappa_{2}\right.$, Table 6) is estimated to be $7.1(\mathrm{p}$-value $=0.01)$, providing strong evidence that an increase in load leads to an early discharge. In particular, a $10 \%$ increase in load leads to an early discharge by 0.7 days on average. When we examine the effect of early discharges on the post-discharge mortality rate, we find that the coefficient $\eta_{4}$ has an odds ratio that is close to 1 (coefficient $=0.13$, Odds Ratio $=1.14$ ), suggesting a small increase in odds of mortality associated with an early discharge. However, the probability of a 30-day post-discharge mortality of any randomly selected patient

\footnotetext{
${ }^{12}$ We thank the DE for pointing out how staffing difference between weekdays and weekends could impact patient length of stay.

${ }^{13}$ The increase in probability was estimated using $\frac{\partial p}{\partial O V E R W O R K}=\mu_{2} p(1-p)$ with the average mortality rate of $p=0.068$.
} 
is less than $1 \%$. Consequently, the corresponding increase in odds by a factor of 1.14 due to an early discharge by a day, is small (less than 1 in 1000 cases is associated with an early discharge induced mortality). Furthermore, early discharges by more than a day would require the load to increase by more than $13 \%$. Such variations of the load above the mean of 0.78 are infrequent, as indicated by the low standard deviation. Thus, this effect is statistically significant, as hypothesized, but small in absolute magnitude.

In summary, we find two effects related to quality. First, overwork leads to an increase in the in-hospital mortality rate. Second, increased levels of load lead to early discharges, which in turn is associated with a small increase in the post-discharge mortality rate.

\section{Discussions and Future Research}

Prior research has assumed that the service rate in a service operations facility is independent of the level of load on the system. We present a model of service worker productivity that includes the effect of load and (over time) the subsequent overwork on service rates. We also consider the quality implications of variable service rates. For the two vastly different services in our study, we find that resources in hospitals are sensitive to their levels of load and that service workers can adapt to system needs by expending more effort to increase the service rate as required.

Various researchers (e.g. Dranove 2002) have reported that hospitals, like most financially oriented entities, have an incentive to increase profits when possible. For instance, Friedman and Pauly (1983) and Anderson and Steinberg (1984) have shown that hospitals exhibit a profit-maximizing response to changes in reimbursement terms. In the US, the diagnosis related group (DRG) for the diagnosis of the patient at the time of discharge determines the amount that the hospital is paid (Fed- 
eral Trade Commission and Department of Justice Report 2004). ${ }^{14}$ Hospitals receive this payment regardless of the realized cost of care; thus, each additional increase in length of stay beyond the standard expected stay generates zero or minimum marginal revenues. ${ }^{15}$ Under DRG-based payment, hospitals have an incentive to increase admissions (Friedman and Pauly 1983). At high levels of load, a hospital's ability to admit new revenue generating patients is reduced. In this paper, we do not empirically examine the underlying incentive schemes. However, it is conceivable that a hospital facing a high load may have a financial incentive to reduce the duration of stay for patients who can be safely discharged earlier, in order to make room for new admissions. As we demonstrate from our analysis, this could have negative consequences for the patient's quality of care. Any potentially conflicting economic and service quality incentives need to be further examined empirically. With the advent of new policy changes in reimbursements to hospitals such as pay for performance (where a hospital's reimbursement is tied directly to its outcomes), there is greater need to empirically examine the role of hospital operations on its financial health.

We also show that increases in productivity cannot be sustained over a long period of time. Traditional wisdom has been that services should operate at close to full utilization to take advantage of capacity costs. However, sustained levels of high utilization results in overwork and the resultant decrease in productivity may offset any cost savings from operating at high utilization. In many service operations, the impact of high system load on the quality of service is a significant consideration for service managers. In our analysis of cardiothoracic surgery, we find that overwork increases the likelihood of mortality - a finding that is consistent with prior medical literature. We also identify a small decline in service quality which is correlated with

\footnotetext{
${ }^{14}$ Each DRG has a payment weight assigned to it, which reflects the average cost of treating patients in that DRG.

${ }^{15}$ Certain hospitals receive an adjusted payment in excess of the standard DRG amount. Actual outlier adjustments are specific to a DRG and are typically made to teaching hospitals and hospitals that treat a disproportionate number of low income patients.
} 
an accelerated service rate (or early discharge).

We found the area of hospital operations to be a fruitful area to create a framework of service worker productivity. Future research needs to investigate if and how our findings apply to other services. For example, the impact of load (and queue length) on quality of inspections is of paramount importance in areas such as airport baggage screening (Jacobson et al, 2003) and in port security (Bakshi et al, 2008). One could also expect the effect of load and overwork to impact quality of service in a variety of applications including call centers and financial services (e.g. loan underwriting). Based on our interactions with the medical and business professionals at our research site we also encountered a great interest to explore questions beyond the research presented in this paper. Future research could also look at accounting for other factors, beyond those used in the study that could affect the case severity. An extreme application in which the interaction between work-load, fatigue, and early discharge is especially of interest to the medical community is the Intensive Care Unit (ICU), and we hope that future research can extend our analysis to this important area of healthcare operations.

\section{References}

Aiken, L. H., S. P. Clarke, D. M. Sloane, J. Sochalski, J. H. Silber. 2002. Hospital nurse staffing and patient mortality, nurse burnout, and job dissatisfaction. Journal of the American Medical Association 288 1987-1993

Anderson, G., E. Steinberg. 1984. Hospital Readmissions in the Medicare Population. New England Journal of Medicine 311 1349-1353

Bakshi, N., N. Gans, S. E. Flynn. 2008. Measuring the Operational Impact of Container Security Initiative. Wharton School Working Paper.

Berk, E. and K. Moinzadeh. 1998. The Impact of Discharge Decisions on Health 
Care Quality. Management Science 44 (3)

Bertsekas, D. P. Dynamic Programming and Optimal Control. 2000. Athena Scientific.

Cakir, A., D. J. Hart, T. F. Stewart. 1980. Visual Display Terminals: A Manual Covering Ergonomics, WorkPlace Design, Health and Safety, Task Organization, John Wiley \& Sons, Inc., New York, NY

Caldwell, J.A. 2001. The impact of fatigue in air medical and other types of operations: a review of fatigue facts and potential countermeasures. Air Med J. 20(1) 25-32

Cameron, A. C. and P. K. Trivedi. 2005. Microeconometrics: Methods and Applications. Cambridge University Press.

Crabill, T. B. 1972. Optimal Control of a Service Facility With Variable Exponential Service Times and Constant Arrival Rate. Management Science 18 (9)

Department of Justice and Federal Trade Commission. 2004. Improving Health Care: A Dose of Competition. A Report by the Federal Trade Commission and the Department of Justice. July 2004.

Dawson, Drew and Phyllis Zee. 2005. Work Hours and Reducing Fatigue-Related Risk: Good Research vs Good Policy. JAMA Vol 294 No 9.

Dranove, D. 2002. The Economic Evolution of American Health Care: From Marcus Welby to Managed Care. Princeton University Press.

EuroSCORE model. 2007. Retrieved April 23, 2007. http://www.euroscore.org Friedman, B., M. V. Pauly. 1983. A New Approach to Hospital Cost Functions and Some Issues in Revenue Regulation. Health Care Financing Review 4(3) 105-114

Fuloria, P. C., S. A. Zenios. 2001. Outcomes-adjusted reimbursements in a health care delivery system. Management Science 46(10) 1317-1366

Gaba, D. M., S. K. Howard. 2002. Fatigue Among Clinicians and The Safety of Patients. New England Journal of Medicine 347(16)

Gerchak, Y., D. Gupta, M. Henig. 1996. Reservation planning for elective surgery 
under uncertain demand for emergency surgery. Management Science, 42(3) 321-334 George, J. M., J. M. Harrison. 2004. Dynamic Control of a queue with adjustable Service Rate. Operations Research 49(5) 720-731

Green, L. V. 1984. A multiple dispatch queueing model of Police Patrol Operations. Management Science Vol 30 No 6.

Green, L. V. 2004. Capacity planning in hospitals. Handbook of Operations Research/Management Science Applications in Health Care, Kluwer Academic Publishers

Green, L. V., J. Meissner. 2002. Developing insights for nurse staffing. Columbia Business School, Working Paper

Green, L. V., V. Nguyen. 2001. Strategies for cutting hospital beds: the impact on patient service. Health Services Research, 36(2) 421-442

Green, L. V., S. Savin, B. Wang. 2006. Managing patient service in a diagnostic medical facility. Operations Research 54(1) 11-25

Huang, X. A. 1995. A planning model for requirement of emergency beds. Journal of Mathematics Applied in Medicine Biology, 12 345-353

Jacobson, Sheldon H., J. Virta, J. M. Bowman, J. E. Kobza and J. J. Nestor. 2003. "Modeling Aviation Baggage Screening Security Systems: A Case Study," IIE Transactions , Vol. 35, No. 3, pp. 259-269

Kurki, T. S. 2002. Prediction of Outcome in Cardiac Surgery. Mount Sainai Journal of Medicine $\mathbf{6 9}(1,2)$

Kwak, N., C. Lee. 1997. A linear programming model for human resource allocation in a health-care organization. Journal of Medical Systems 21 129-140 Nashef, S. A., F. Roques, B. G. Hammill, E. D. Peterson, P. Michel, F. L. Grover, R. K. Wyse, T. B. Ferguson. 2002. Validation of European System for Cardiac Operative Risk Evaluation (EuroSCORE) in North American cardiac surgery European Journal of Cardiothoracic Surgery 22 101- 105

Needleman, J., P. I. Buerhaus, M. Stewart, K. Zelevinksy, S. Mattke. 2006. Nurse 
Staffing In Hospitals: Is There A Business Case For Quality? Health Affairs 25(1) 204-211

Newhouse, J. P. 1996. Reimbursing health plans and health providers: Efficiency production versus selection. J. Econom. Literature 34 1286-1263

Powell, S. G., K. L. Schultz. 2004. Throughput in Serial Lines with State-Dependent Behavior. Management Science 50(8) 1095-1105

Reinhart, E., P. S. Husse, G. F. Anderson. 2004. U.S. Health Care Spending in an International Context. Health Affairs 23(3) 10-25

Setyawati, L. 1995. Relation between feelings of fatigue, reaction time and work productivity. J Hum Ergol (Tokyo). 24(1)129-35

V. Smith-Daniels, S. B. Schweikhart, D. E. Smith-Daniels. 1988. Capacity management in health care services: review and future research directions. Decision Sciences $19889-919$

Schultz, K. L., D. C. Juran, J. W. Boudreau. 1999. The Effects of Low Inventory on the Development of Productivity Norms. Management Science 45(12) 1664-1678

Schultz, K. L., D. C. Juran, J. W. Boudreau, J. O. McClain, L. J. Thomas. 1998. Modeling and Worker Motivation in JIT Production Systems. Management Science 44(12) 1595-1607

Scott, L. D., A. E. Orgers, W. Hwang, Y. Zhang. 2006. Effects of Critical Care Nurses' Work Hours on Vigilance and Patient's Safety. American Journal of Critical Care $\mathbf{1 5}(1)$

Jacobson, S. H., J. Virta, J. M. Bowman, J. E. Kobza and J. J. Nestor. 2003. Modeling Aviation Baggage Screening Security Systems: A Case Study IIE Transactions Vol. 35, No. 3, pp. 259-269, March 2003.

Stidham, S., R. R. Weber. 1989. Monotonic and Insensitive Optimal Policies for Control of Queues with Undiscounted Costs Operations Research 87(4)

Tanabe, S., S. Nishihara. 2004. Productivity and fatigue. Indoor Air Col 14(suppl 7) $126-133$ 
Toumpoulis, I. K., C. E. Anagnostopoulos, D. G. Swistel, J. J. DeRose, Jr. 2005. Does EuroSCORE predict length of stay and specific postoperative complications after cardiac surgery? Eur Journal of Cardiothoracic Surgery 27 128-133

\section{Tables and Figures}

Figure 2: Effect of Load and Overwork on Service Time

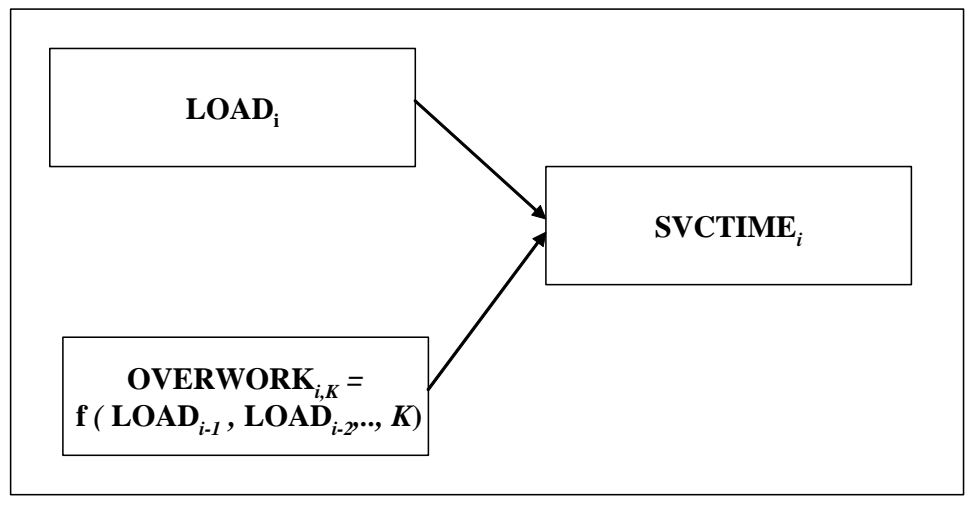

Figure 3: Effect of Load Overwork and Service Time on Quality

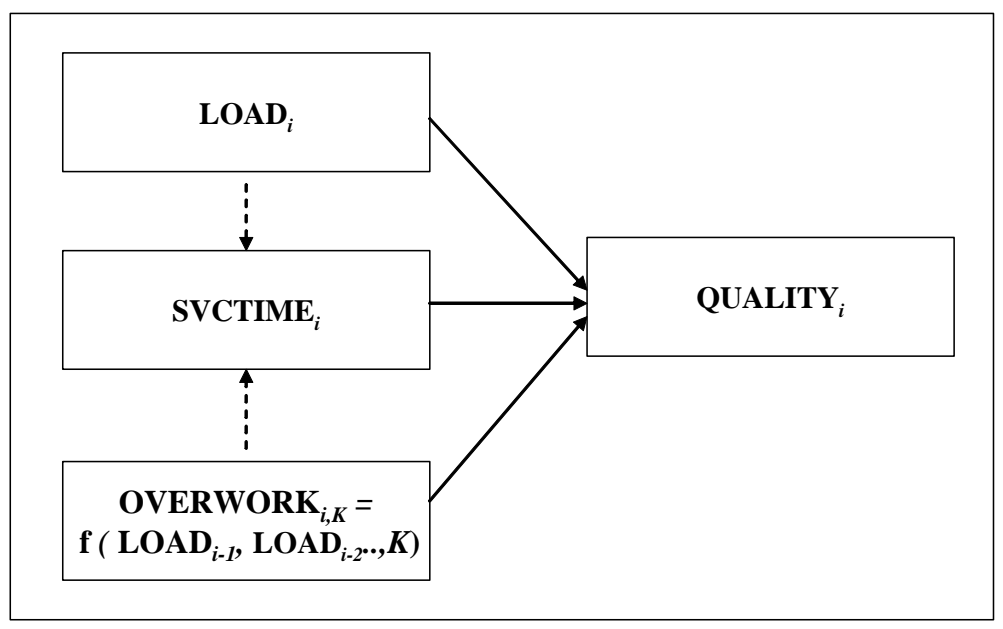

Table 1: Transport Descriptive Statistics

\begin{tabular}{llll}
\hline Measure & Mean & Standard Deviation & Median \\
\hline SVCTIME (minutes) & 12.6 & 7.75 & 10.35 \\
LOAD & 0.755 & 0.21 & 0.73 \\
OVERWORK $_{\mathbf{K}=4}$ & 0.001 & 0.21 & 0.02 \\
\hline
\end{tabular}


Table 2: Effect of Load and Overwork on Transport Time

\begin{tabular}{|c|c|c|c|}
\hline \multirow{2}{*}{$\frac{\text { Coefficient }}{\text { Intercept }}$} & Model 1 & \multicolumn{2}{|c|}{ Model 2} \\
\hline & $\begin{array}{l}2.73 \\
(0.74) * * *\end{array}$ & \multicolumn{2}{|c|}{2.13} \\
\hline $\boldsymbol{\beta}_{2}$ & -0.17 & \multicolumn{2}{|c|}{-0.12} \\
\hline$\beta_{3}$ & $(0.07) * * *$ & \multicolumn{2}{|c|}{0.09} \\
\hline F-Statistic & $4.5(\mathrm{p}<0.01)$ & \multicolumn{2}{|c|}{$\begin{array}{l}(0.05) * * \\
4.5(\mathrm{p}<0.01)\end{array}$} \\
\hline \multicolumn{4}{|c|}{$\begin{array}{l}\text { Standard errors are shown in parentheses. } * * *, * * \text {, and } * \text { denote statistical significance at the } 1 \%, 5 \% \text { and } \\
10 \% \text { confidence levels respectively. Dummy variables for } X_{i} \text { (Appendix) not displayed }\end{array}$} \\
\hline \multicolumn{4}{|c|}{ Table 3: Cardiothoracic Surgery Descriptive Statistics } \\
\hline SVCTIME (davs) & $\frac{1 \text { inedil }}{12.98}$ & 10.69 & 7 \\
\hline LOAD & 0.78 & 0.086 & 0.79 \\
\hline OVERWORK $_{K=7 \text { Davs }}$ & 0.005 & 0.07 & 0.01 \\
\hline MORTALITY & 0.068 & 0.255 & 0 \\
\hline
\end{tabular}

Table 4: Effect of Load, Overwork and Early Week Admission on Patient Length of Stay

\begin{tabular}{llll}
\hline Coefficient & Model 1 & Model 2 & Model 3 \\
\hline Intercept & 2.21 & 2.13 & 2.6 \\
& $(0.09) * * *$ & $(0.09) * * *$ & $(0.07) * * *$ \\
$\gamma_{\mathbf{2}}$ & -2.07 & -2.08 & -0.58 \\
& $(0.26) * * *$ & $(0.26) * * *$ & $(0.11) * * *$ \\
$\gamma_{3}$ & 2.27 & 2.28 & \\
& $(0.36) * * *$ & $(0.36) * * *$ & \\
$\gamma_{4}$ & -0.09 & & -0.09 \\
F-Statistic & $(0.03) * * *$ & & $(0.03) * * *$ \\
\hline
\end{tabular}

Standard errors are shown in parentheses. $* * *, * *$, and $*$ denote statistical significance at the $1 \%, 5 \%$ and $10 \%$ confidence levels respectively. Dummy variables for $Y_{i}$ (Appendix) not displayed

Table 5: In-Hospital Mortality Results

\begin{tabular}{llll}
\hline Coefficient & Estimate & Odds Ratio & $\begin{array}{l}\text { 95\% Wald } \\
\text { Confidence Limit }\end{array}$ \\
\hline Intercept & $-1.3(0.83)^{*}$ & $0.002,0.127$ \\
$\boldsymbol{\mu}_{\mathbf{2}}$ & $-4.09(3.52)$ & 0.017 & $1.89,622$ \\
$\boldsymbol{\mu}_{\mathbf{3}}$ & $3.53(1.4)^{* * *}$ & 34.37 & \\
\hline Likelihood Ratio $\left(\chi^{2}\right)$ & $234.6(\mathrm{p}-\mathrm{value}<0.0001)$ & \\
\hline Standard errors are shown in parentheses. $* * *, * *$, and $*$ denote statistical significance at the 1\%, 5\% and \\
10\% confidence levels respectively. Dummy variables for $Z_{i}$ (Appendix) not displayed
\end{tabular}


Table 6: Early Discharges Resulting from Increased Load

\begin{tabular}{ll}
\hline Coefficient & Estimate \\
\hline Intercept & $1.97(0.67)$ \\
$\mathbf{K}_{\mathbf{2}}$ & $7.1(1.4) * * *$ \\
F-Statistic & $5.57(\mathrm{p}<0.01)$ \\
\hline Standard errors are shown in parentheses. ${ }^{* * *}, * *$, and $*$ denote statistical significance at the $1 \%, 5 \%$ and \\
10\% confidence levels respectively
\end{tabular}

Table 7: Post-Discharge Mortality Results

\begin{tabular}{llll}
\hline Coefficient & Estimate & Odds Ratio & $\begin{array}{l}\text { 95\% Wald Confidence } \\
\text { Limit }\end{array}$ \\
\hline Intercept & $-3.31(2.62)$ & & \\
$\boldsymbol{\eta}_{\mathbf{2}}$ & $-4.02(3.38)$ & 0.018 & $(0.001,13.606)$ \\
$\boldsymbol{\eta}_{\mathbf{3}}$ & $9.2(5.44)$ & $>999$ & $(0.111,9.19)$ \\
$\boldsymbol{\eta}_{\mathbf{4}}$ & $0.131(0.04) * * *$ & 1.14 & $(1.043,1.246)$ \\
\hline Likelihood Ratio $\left(\boldsymbol{\chi}^{2}\right)$ & $48.1(\mathrm{p}<0.01)$ & & \\
\hline
\end{tabular}

Standard errors are shown in parentheses. $* * *, * *$, and $*$ denote statistical significance at the $1 \%, 5 \%$ and $10 \%$ confidence levels respectively. Dummy variables for $Z_{i}$ (Appendix) not displayed 